OPEN ACCESS

Edited by:

Jane Ward,

Rothamsted Research,

United Kingdom

Reviewed by:

Manoj Prasad,

National Institute of Plant Genome

Research (NIPGR), India

Atsushi Fukushima,

RIKEN, Japan

*Correspondence:

Xiaoming Wang

wang_xm635@126.com

Zhihai Zhao

zhaozhihai58@163.com

†These authors have contributed equally to this work

Specialty section: This article was submitted to

Plant Metabolism

and Chemodiversity,

a section of the journal

Frontiers in Plant Science

Received: 08 February 2021 Accepted: 20 May 2021 Published: 27 July 2021

Citation:

Wei W, Li S, Wang Y, Wang B,

Fan $G$, Zeng $Q$, Zhao $F, X u C$, Zhang $X$, Tang $T$, Feng $X$, Shi J, Shi $G$ Zhang W, Song G, Li H, Wang F, Zhang $Y$, Li X, Wang $D$, Zhang $W$, Pei J, Wang $X$ and Zhao Z (2021) Metabolome-Based Genome-Wide Association Study Provides Genetic Insights Into the Natural Variation of Foxtail Millet.

Front. Plant Sci. 12:665530. doi: 10.3389/fpls.2021.665530

\section{Metabolome-Based Genome-Wide Association Study Provides Genetic Insights Into the Natural Variation of Foxtail Millet}

\author{
Wei Weit, Shuangdong $\mathrm{Li}^{1+}$, Yixiang Wang ${ }^{2 t}$, Bin Wang', Guangyu Fan', Qisen Zeng', \\ Fang Zhao' ${ }^{1}$, Congping $\mathrm{Xu}^{2}$, Xiaolei Zhang ${ }^{1}$, Tang Tang ${ }^{2}$, Xiaolei Feng ${ }^{1}$, Jian Shi', \\ Gaolei Shi', Weiqin Zhang ${ }^{2}$, Guoliang Song ${ }^{1}$, Huan $L^{2}{ }^{2}$, Feng Wang ${ }^{1}$, Yali Zhang ${ }^{1}$, \\ Xinru Li ${ }^{1}$, Dequan Wang ${ }^{1}$, Wenying Zhang ${ }^{1}$, Jingjing Pei ${ }^{1}$, Xiaoming Wang ${ }^{1 *}$ and \\ Zhihai Zhao ${ }^{\text {** }}$ \\ ${ }^{1}$ Institute of Millet, Zhangjiakou Academy of Agricultural Science, Zhangjiakou, China, ${ }^{2}$ Wuhan Metware Biotechnology Co., \\ Ltd., Wuhan, China
}

The plant metabolome is considered as a bridge between the genome and the phenome and is essential for the interaction between plant growth and the plant environment. Here, we used the liquid chromatography-tandem mass spectrometry method to perform a widely targeted metabolomics analysis of 150 millet germplasm and simultaneous identification and quantification of 330 annotated metabolites. Comparing the metabolic content of different millets revealed significant natural variation of both primary and secondary metabolites, including flavonoids, phenolamides, hydroxycinnamoyl derivatives, nucleotides, and lipids, in the millets from India and the north and south of China; among them, some of the flavonoids are the most prominent. A total of 2.2 TB sequence data were obtained by sequencing 150 accessions of foxtail millet using the lllumina platform. Further digging into the genetic basis of metabolites by mGWAS analysis found that cyanidin 3-O-glucoside and quercetin O-acetylhexside are concentratedly located at $43.55 \mathrm{Mb}$ on chromosome 5 and $26.9 \mathrm{Mb}$ on chromosome 7, and two Lc were mined as candidate genes, respectively. However, the signals of luteolin 7-O-glucoside and kaempferol 3-O-glucoside were also detected at $14.36 \mathrm{Mb}$ on chromosome 3 , and five glycosyltransferase genes on this loci were deemed to regulate their content. Our work is the first research to use mGWAS in millet, and it paves the way for future dissection of complex physiological traits in millet.

Keywords: foxtail millet, metabolic profiling, flavonoids, phenolamides, natural variation, mGWAS

\section{INTRODUCTION}

As a healthy food material for the development of functional foods and natural antioxidant resources, foxtail millet (Setaria italica) is an important grain crop. It is characterized by adaptation to unfavorable ecological conditions, such as irregular and untimely rainfall, high-salinity soil, and high temperatures (Doust et al., 2009; Aidoo et al., 2016). The foxtail millet has been domesticated by humans from its wild species, green foxtail (Setaria viridis), in Northern China 
for over 8000 years (Barton et al., 2009), and it is widely cultivated as a dietary staple in the world, particularly in China and India with their rich and diverse germplasm (Bettinger et al., 2010; Lata et al., 2013). The metabolome is playing an increasingly important role in providing plants with environmental adaptability, Anthocyanins are commonly helped plants in response to different abiotic stresses, including drought, salinity, excess light, and extreme temperatures of hot and cold (Garriga et al., 2014; Kovinich et al., 2015; Miki et al., 2015). Investigation of the metabolome in rice and maize core collections has demonstrated significant natural variation of secondary metabolites, such as flavonoids, phenolamides, hydroxycinnamic acid derivatives, and terpenoids, in these species (Chen et al., 2014; Dong et al., 2014; Wen et al., 2014). For example, flavonoids, such as flavone mono- $C$-hexosides, malonylated flavone $O$-hexosides, and flavone 5-O-glycosides, accumulated in relevantly lower levels in the rice subspecies japonica than that in indica, while phenolamides, such as $N$ $p$-coumaroyl spermidine and $N$-sinapoyl agmatine/putrescine, accumulated at significantly higher levels in japonica than in indica (Dong et al., 2014; Wen et al., 2014). Among them, the natural variation of flavone $\mathrm{O}$-glycosides has been demonstrated to determine the variation of UV tolerance in rice accessions (Peng et al., 2017). However, investigations of natural variation in metabolic traits, as well as their relationship with complex agronomic traits in foxtail millet are lacking.

In recent years, with the rapid development of gene chip and genome sequencing technology, high-quality genetic linkage maps and genome sequence information has been obtained in most crops. At the same time, there has been a continuous improvement in plant metabolite detection technology allowing more metabolites can be detected (Chen et al., 2013; AlvarezRivera et al., 2019; Ballesteros-Vivas et al., 2019). Combine these two aspects, produced the genome-wide association study, and metabolomics analysis (mGWAS) has become a powerful tool of forwarding genetics, which is suitable for exploring the genetic and biochemical basis of plant metabolism; thus far, it has been applied on metabolite structure analysis and functional genomics. Through mGWAS analysis of 805 metabolic traits in wheat, 1098 marker-metabolite associations were obtained, 26 candidate genes were unearthed, and the in vitro enzyme activity experiment revealed the main modification pathways of flavonoids in wheat (Chen et al., 2020). In the highland barley (Qingke), mGWAS was used to explain the accumulation of phenylpropane metabolic pathways in the high-level UV-B process of highland barley for its two aspects (being constitutive and inducible), and the genetic basis was studied (Zeng et al., 2020). In rice, the combined analysis of mGWAS and pGWAS proved that metabolic traits and phenotypes are interlinked, and new candidate genes for grain color and size were discovered. It provides a powerful strategy for interactive functional genomics and metabolomics in plants, especially the cloning of minor QTLs for complex phenotypic traits (Chen et al., 2014).

In this study, we collected a total of 150 diverse foxtail millet varieties from a majority of the tropical and temperate regions. We performed profiling of comprehensive metabolic and comparative analysis of metabolites at the primary and secondary metabolites in the millet population. Flavonoids, phenolamides, hydroxycinnamoyl derivatives, nucleotides, and lipids have an obvious natural variation of millet leaves in India and the north and south of China. Then, we used mGWAS to conduct preliminary genetic research on important flavonoid aglycone, identified two anthocyanin regulatory $L c$ proteins that affect the content of cyanidin 3-O-glucoside and quercetin $O$-acetylhexoside, five glycosyltransferase genes that regulate the synthesis of luteolin 7-O-glucoside and kaempferol 3-Oglucoside. The natural variation of metabolites may provide a theoretical basis for revealing that millet has different growth and development environments.

\section{MATERIALS AND METHODS}

\section{Plant Materials}

The 150 millet varieties used in this study were taken from a collection of cultivated germplasm (Supplementary Table 1). The leaves at the five-leaf stage were collected from those varieties. The populations were evaluated in natural field conditions in the experimental farm of Zhangjiakou Academy of Agricultural Science, Zhangjiakou, China $\left(144^{\circ} 88^{\prime} \mathrm{N}, 40^{\circ} 77^{\prime} \mathrm{E}\right)$, in a randomized complete block design. In each line was planted in a two-row plot of $1.5 \mathrm{~m}$ length with the spacing of $0.1 \mathrm{~m}$ between plants and $0.2 \mathrm{~m}$ between rows. Field management, including irrigation, fertilization, weeding, and pest control, followed the standard agricultural practices in millet production. Samples were taken from three different plants per line and pooled for each biological replicate. Two biological replicate leaf samples obtained from the same accessions were used for the GWAS. All the samples were harvested at 10:00-12:00 on that day, placed in liquid $\mathrm{N} 2$ immediately, and stored at $-70^{\circ} \mathrm{C}$ until vacuum freezedrying.

The 150 accessions used in this study were characterized by whole genome re-sequencing. DNA was isolated from young leaves and sequencing libraries with short inserts were constructed following the manufacturer's instructions (Illumina). The samples were sequenced on an Illumina HiSeq 4000 platform. To retain reads of high quality, reads with fewer than 5\% $\mathrm{N}$ (missing) bases and with fewer than 50\% of bases of base quality $<5$ were deemed as cleaned reads. All other reads were discarded.

\section{Chemicals}

All the chemicals were of analytical reagent grade. Gradientgrade methanol, acetonitrile, and acetic acid were purchased from Merck Company, Germany. ${ }^{1}$ The water was doubly deionized with a Milli-Q water purification system (Millipore, Bedford, MA, United States). Authentic standards were purchased from ANPEL, Shanghai, China, ${ }^{2}$ BioBioPha Co., Ltd., ${ }^{3}$ and SigmaAldrich, United States. ${ }^{4}$ Standards stock solutions were prepared

\footnotetext{
${ }^{1}$ http://www.merck-chemicals.com

${ }^{2}$ http://www.anpel.com.cn/cnw

${ }^{3}$ http://www.biobiopha.com

${ }^{4}$ http://www.sigmaaldrich.com
} 
using water, methanol, and/or dimethyl sulfoxide (DMSO) as the solvent and stored at $-20^{\circ} \mathrm{C}$. Combined standard solutions of chemicals were prepared just before use by mixing individual stock solutions and diluting these mixtures with $70 \%$ aqueous methanol.

\section{Sample Preparation and Extraction}

The freeze-dried leaves were crushed using a mixer mill (MM 400, RETSCH) with zirconia beads for $1.5 \mathrm{~min}$ at $30 \mathrm{~Hz}$. A $100 \mathrm{mg}$ mass of powder was weighed and extracted overnight at $4^{\circ} \mathrm{C}$ with $1.0 \mathrm{ml}$ of $70 \%$ aqueous methanol. Following centrifugation at $10,000 \times g$ for $10 \mathrm{~min}$, the extracts were filtered (SCAA104, $0.22 \mu \mathrm{m}$ pore size; ANPEL, Shanghai, China ${ }^{5}$ ) before LCMS analysis.

\section{LC-MS Conditions}

The sample extracts were analyzed using an LC-ESI-MS/MS system (HPLC, Shim-pack UFLC SHIMADZU CBM30A system $^{6}$; MS, Applied Biosystems $6500 \mathrm{Q}^{\mathrm{TRAP}}{ }^{7}$ ). The analytical conditions were as follows, HPLC: column, Waters ACQUITY UPLC HSS T3 C18 (1.8 $\mu \mathrm{m}, 2.1 \mathrm{~mm} \times 100 \mathrm{~mm})$; solvent system, water ( $0.04 \%$ acetic acid): acetonitrile ( $0.04 \%$ acetic acid); gradient program, 100:0 V/V at $0 \mathrm{~min}, 5: 95 \mathrm{~V} / \mathrm{V}$ at $10.0 \mathrm{~min}$, $5: 95 \mathrm{~V} / \mathrm{V}$ at $11.0 \mathrm{~min}, 95: 5 \mathrm{~V} / \mathrm{V}$ at $11.1 \mathrm{~min}, 95: 5 \mathrm{~V} / \mathrm{V}$ at $15.0 \mathrm{~min}$; flow rate, $0.35 \mathrm{ml} / \mathrm{min}$; temperature, $40^{\circ} \mathrm{C}$; and injection volume: $5 \mu$ l. The effluent was alternatively connected to an ESI-triple quadrupole-linear ion trap (Q TRAP)-MS.

LIT and triple quadrupole (QQQ) scans were acquired on a triple quadrupole-linear ion trap mass spectrometer (Q TRAP) using an API 6500 Q TRAP LC/MS/MS System, which was equipped with an ESI Turbo Ion-Spray interface operated in a positive ion mode and controlled by Analyst 1.6.3 software (AB Sciex). The ESI source operation parameters were as follows: ion source, turbo spray; source temperature $550^{\circ} \mathrm{C}$; ion spray voltage (IS) $5500 \mathrm{~V}$; ion source gas I (GSI), gas II (GSII), and curtain gas (CUR) were set at 55, 60, and 30.0 psi, respectively; and the collision gas (CAD) was high. Instrument tuning and mass calibration were performed with 10 and $100 \mu \mathrm{mol} / \mathrm{L}$ polypropylene glycol solutions in QQQ and LIT modes, respectively. The QQQ scans were acquired as MRM experiments with the collision gas (nitrogen) set to 5 psi. The DP and CE for individual MRM transitions were performed with further DP and CE optimization. A specific set of MRM transitions was monitored for each period according to the metabolites that were eluted within this period.

Obtaining the highly reproducible metabolite signals with the production spectra. Based on the annotation, commercially available standards were purchased and analyzed using the same profiling procedure as the extracts.

\section{Genome-Wide Association Analyses}

Only SNPs with minor allele frequencies $\mathrm{R} 0.05$ and with the number of varieties having the minor allele in a (sub)

\footnotetext{
${ }^{5}$ http://www.anpel.com.cn/

${ }^{6}$ www.shimadzu.com.cn/

${ }^{7}$ www.appliedbiosystems.com.cn/
}

population R6 were used to carry out GWAS. After obtaining the metabolome data, combined with the genotypes of 150 related populations, mGWAS analysis was first performed on the related population data. GWAS analysis used the compressed mixed linear model (cMLM), which integrates metabolic phenotype, genotype, population structure, and genetic relationship, to analyze, obtaining the association with each genotype (SNP) and phenotype $P$-value. After Bonferroni correction $(P=0.05 / 1,963,697)$, it was used to correct the threshold of localization, where the significant threshold of mGWAS after correction is $P \leq 1.8 \times 10^{-6}$, and thus to locate the area significantly associated with the metabolic phenotype. Then, we used R to draw Manhattan graph and Q-Q graph in order to visually show the significant associated area and model effect.

\section{Statistical Analysis}

Principal component analysis (PCA) was performed with $\log 2$ transformed metabolite data to improve the normality. For hierarchical clustering analysis (HCA) in the study of developmentally controlled accumulation and natural variation of metabolites, metabolite data were firstly $\log 2$ transformed, followed by a min-max normalization. To construct the neighbor-joining tree, the data matrix was generated from 150 millet varieties and 1335 detected metabolites, which represented the contents of each metabolite in different populations. The pairwise population distance was used to construct a neighborjoining tree using the software PHYLIP (version 3.69). The software TreeView and MEGA7 were used for visualizing the phylogenetic tree. For HCA, the "heatmap.2" function in the "gplot" R-package was utilized to generate the heatmap, and various $\mathrm{R}$ programming tools were used to plot data. ${ }^{8}$ Identification of differential accumulation of metabolites between different tissue/varieties was determined by partial least squaresdiscriminate analysis (PLS-DA) with the VIP values (Variable Importance for the Projection) $\geq 0.8$, followed by both ANOVA $(p \leq 0.05)$ and fold-change $(\geq 1.5$ or $\leq 0.67)$. PCA and PLS-DA were performed with SIMCA-P version 14.0.

\section{$\boldsymbol{F}_{\text {ST }}$ Analysis}

Developed as a special case of Wright's $F$ statistics, the fixation index $\left(F_{\mathrm{ST}}\right)$ is one of the most commonly used statistics in population genetics, and it is a measure of population differentiation due to genetic structure, which is frequently estimated from genetic polymorphism data, such as SNPs (Holsinger and Weir, 2009). Estimates of $F_{\text {ST }}$ can identify regions of the genome that have been the target of selection, and comparisons of $F_{\mathrm{ST}}$ from different parts of the genome can provide insights into the demographic history of populations. For selection signature analysis, SNPs of the whole genome were used, and a sliding window approach (100-kb windows sliding in 10$\mathrm{kb}$ steps) was employed to quantify genetic differentiation $\left(F_{\mathrm{ST}}\right)$ for Indian and Northern Chinese accessions using the VCFtools software. Windows with $F_{\mathrm{ST}}$ values exceeding an empirical $F_{\mathrm{ST}}$ cutoff (top 5\%) were regarded as highly differentiated regions.

\footnotetext{
${ }^{8}$ http://cran.r-project.org/web/packages/gplots/index.html
} 
Windows separated by less than $200 \mathrm{~kb}$ were merged into a single non-overlapping region.

\section{Phylogenetic Analysis}

The amino acid sequences of reported genes including $L c$ transcription factors and glycosyltransferase were obtained from NCBI according to their accession numbers. ${ }^{9}$ Candidate gene information in this study was obtained from the draft assembly of the millet genome (Bennetzen et al., 2012). The alignment of amino acid sequences was performed using ClustalW bundled in MEGA 5, and neighbor-joining trees were constructed using MEGA 5 software with all default parameters. The reliability of the reconstructed tree was evaluated using a bootstrap test with 1000 replicates.

\section{qRT-PCR Analysis}

To validate the RNA-seq output, seven candidate genes were selected and analyzed by qRT-PCR. The total RNA was extracted from selected samples using $1.5 \times$ CTAB buffer. The qRT-PCR was performed on a 7500 Fast Real-Time PCR System (AB Ltd.) using the SuperReal PreMix Plus (SYBR Green) kit (Tiangen Biotech Co., Ltd., Beijing, China) according to the manufacturer's instructions. The PCR was executed under the following process: $95^{\circ} \mathrm{C}$ for $5 \mathrm{~min}$ for pre-degeneration, then 35 cycles of $94^{\circ} \mathrm{C}$ for $30 \mathrm{~s}, 56^{\circ} \mathrm{C}$ for $30 \mathrm{~s}$, and $72^{\circ} \mathrm{C}$ for $90 \mathrm{~s}$. Each experiment was performed in triplicate for biological and technical repeats. The sequences of primers for qRT-PCR are listed in Supplementary Table 13.

\section{RESULTS}

\section{Metabolic Characterization of Foxtail Millet}

To obtain a global view of the metabolic variation in foxtail millet cultivars, we performed metabolic profiling analyses with leaves at the five-leaf stage from 150 accessions using a previously reported broadly targeted liquid chromatography-tandem mass spectrometry (LC-MS/MS)-based metabolic profiling method (Chen et al., 2013; Supplementary Table 1). By constructing a millet leaf $\mathrm{MS}^{2}$ spectral tag $\left(\mathrm{MS}^{2} \mathrm{~T}\right)$ library, we finally obtained the 1335 highly reproducible metabolite signals with the production spectra (Supplementary Tables 2, 3). Specifically, 133 were identified with the commercial standards, and 197 were putatively annotated as previously described (Dong et al., 2014; Wang et al., 2016; Li et al., 2018). Among which, we identified 155 primary metabolites, including 31 amino acids with their derivatives, 24 nucleotides and their derivatives, 5 carbohydrates, 23 organic acids, and 72 lipids, and a number of secondary metabolites, including 42 flavone $O$-glycosides, 22 flavone $C$-glycosides, 13 flavonol $O$-glycosides, 9 flavanones, 6 anthocyanins, 6 flavonolignans, 12 hydroxycinnamic acid derivatives, 11 hydroxycinnamoyl quinates, 15 phenolamides, 5 phytohormones, 14 benzoic acid derivatives, 4 coumarins, 3 alkaloids, 4 vitamins, and 9 others (Supplementary Figure 1A).

${ }^{9}$ http://www.ncbi.nlm.nih.gov/

\section{Natural Variation of the Metabolome in Foxtail Millet}

To study the natural variation of metabolites among the millet varieties, high-throughput quantification of the 1335 metabolic features was then carried out in the leaf from these varieties for widely targeted metabolic analysis. More than $50 \%$ of the metabolites showed coefficients of variation (CV) greater than 50\% (Supplementary Figure 1B), suggesting their significant variation in millets. Further, the PCA separated the 150 millet accessions into three groups clearly, and a deep insight into the geographical distribution of them revealed that they were mainly represented by the millet varieties from India, the north of China, and the south of China (Figure 1A). The clustering analysis based on the levels of metabolites yielded a similar result and divided these varieties into three major clades from the neighbor-joining tree (Figure 1B), indicating significant metabolic variations in foxtail millets from different geographical locations. When comparing the metabolic accumulation in different groups of millet, fold-change ( $\mathrm{FC} \geq 1.5$ or $\leq 0.67$ ), VIP (VIP $\geq 0.8)$, and $P$-value $(p<0.05)$ were used to evaluation of the metabolite contents and 617 metabolites showed significant variations among the millet varieties (Supplementary Table 4), among which 155 metabolites were annotated. Visualization of the metabolic profile in the millet population by hierarchical cluster analysis revealed a clear phenotypic variation in terms of their abundance among the millets from India, the south and north of China, and the 155 metabolites could be clearly grouped into two main clusters with eight subclusters (Figure 1C). Overall, metabolites in clade 1 , clade 2 , clade 3 , clade 4 , and clade 7 showed significantly higher levels in millet from the south and/or north of China than that in India, and they were mainly represented by flavone $C$-glycosides, flavone $O$-glycosides, flavonol O-glycosides, anthocyanins, hydroxycinnamic acid derivatives, and phenolamides. The metabolites in clade 5 , clade 6 , and clade 8 , however, displayed lower levels in millet from the south and/or north of China when compared to the millet in India, and were mainly represented by $C$-glycosyl-flavone $O$-glycosides, flavone di-O-glycosides, nucleoside phosphate, and glycerophospholipids (Figure 1C), disclosing significant natural variation in both primary and secondary metabolites in millets from different areas. These observations suggested that flavonoid $\mathrm{C} / \mathrm{O}$-glycosides, phenolamides and glycerophospholipids might function as important phytochemical protectants for millets cultivated in different areas.

A deep insight into the differentially accumulated metabolites revealed that, compared to Indian millet, 62 metabolites, including 18 flavone $O$-glycosides, 6 flavone $C$-glycosides, 5 flavanones, 5 flavonols, 6 anthocyanins, 6 hydroxycinnamoyl acid derivatives, 7 phenolamides, and 6 hydroxycinnamoyl quinates, were shown to be significantly higher in millet from the north of China, while 45 metabolites, including 7 flavone $O$-glycosides, 9 flavone $C$-glycosides, 4 flavanone, 7 nucleotides, and 6 glycerophospholipids, were shown to have significantly lower levels (Figure 1C and Supplementary Table 4). Among which, flavonoid $O$-glycosides belonged to different classes, such as naringenin O-glucoside (GZ0896), 

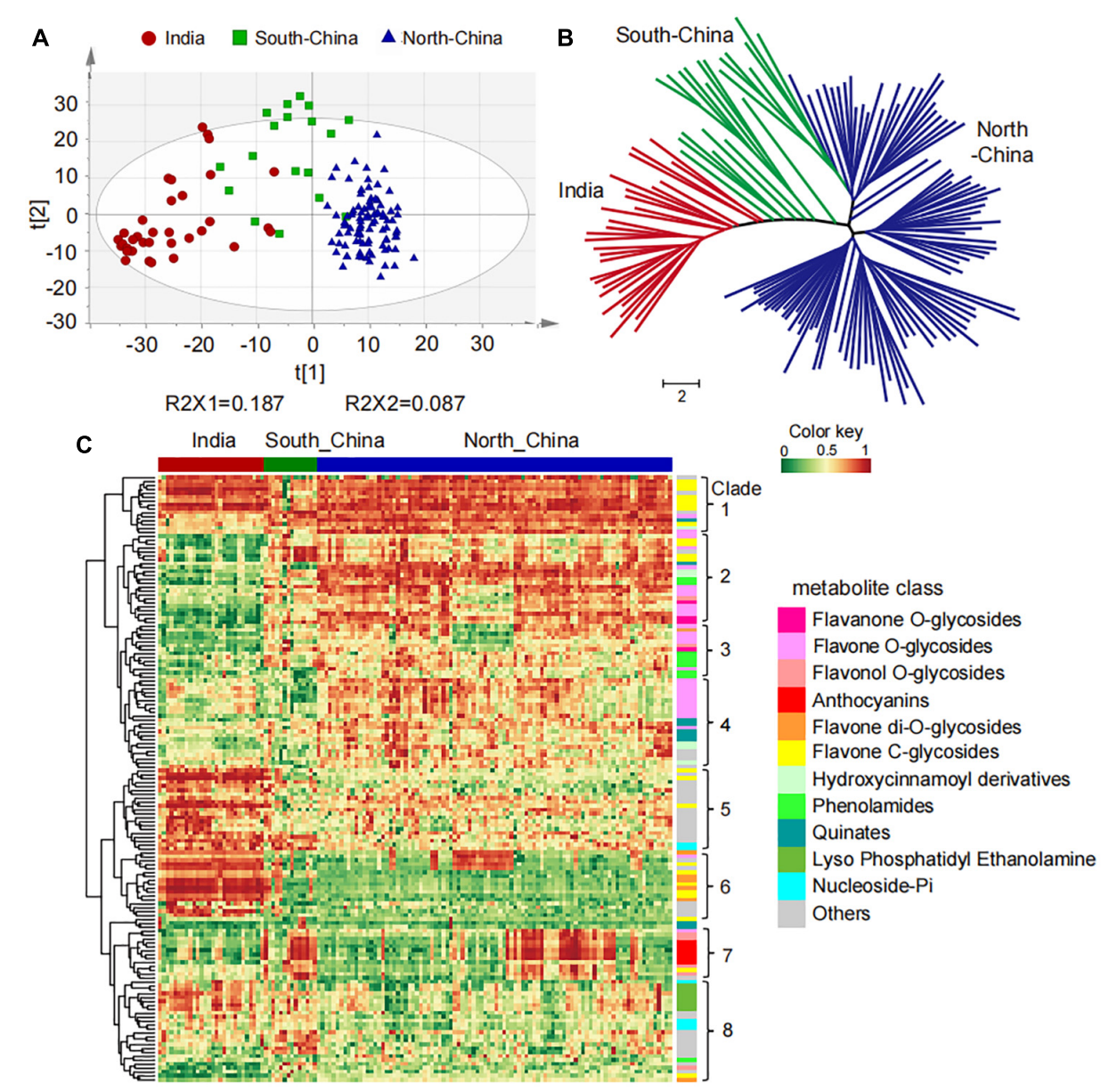

FIGURE 1 | Natural variation and population structure of 150 millet accessions based on metabolic profiling. (A) Score plot of principal component analysis (PCA) for datasets of millet leaf metabolites. The red, green, and blue dots represent samples from India, the south and north of China, respectively. (B) Neighbor-jointing phylogenetic tree of 150 millet accessions with leaf metabolites. Three major clades, represented by India (clade I), South-China (clade II), and North-China (clade III), were identified from the tree. The scale bar indicates the simple matching distance. (C) Heatmap visualization of relative differences of metabolites that showed significant differential accumulation among Indian and Chinese millet accessions. Each millet accession is visualized in a single column and each metabolite is represented by a single row. Data of the content value of each metabolite were normalized to complete linkage hierarchical clustering. Red indicates high abundance, and green indicates low relative metabolic contents.

kaempferol 3-O-glucoside (GZ2519), cyanidin 3-O-glucoside (GZ2192), and tricin 7-O-hexoside (GZ2643) displayed 5.9, 1.8, 5.0 , and 2.0 times over-accumulation in millet from north of China than that from India, respectively (Figure 2A). Flavone $C$-glycoside, hydroxycinnamic acid derivatives, phenolamides, and hydroxycinnamoyl quinates, such as apigenin di-C,Chexoside (GZ2331), 1-O-beta-D-glucopyranosyl sinapate (GZ0627), $N^{\prime}$-p-coumaroyl agmatine (GZ2195), and 3-O-pcoumaroyl quinic acid O-hexoside (GZ0446), also showed significantly higher levels in millet from the north of China with average levels up to $2.5,12.0,3.3$, and 5.8-fold, respectively (Figure 2A). On the contrary, there were significantly lower levels of flavone di-O-glycoside and C-glycosyl-flavone $\mathrm{O}$-glycosides, represented by tricin $\mathrm{O}$-rutinoside (GZ2860) and C-hexosyl-luteolin $O$-feruloylpentoside (GZ2521), and nucleoside phosphate and glycerophospholipids, such as adenosine $5^{\prime}$-monophosphate (GZ1824) and LysoPE 18:2
(GZ3304), in millet from the north of China than that in India, with average decreased levels up to 0.4, 0.6, 0.02, and 0.1-fold (Figure 2B), respectively. A similar result could be observed when comparing the metabolic variation between the millet from India and the south of China. A total of 57 metabolites showed significantly higher, and 46 metabolites showed significantly lower levels in millet from the south of China compared to Indian millet (Figure 2 and Supplementary Table 4).

\section{Genetic Basis of Metabolic Differences in Foxtail Millet}

To uncover the genetic control of natural variation in metabolites, the 150 foxtail millet accessions of mini-core collection of the varieties were subjected to Illumina Hiseq 4000 platform and generated approximately $2.2 \mathrm{~TB}$ of clean data. Pairend reads were mapped against the reference genome of millet genome reference (Bennetzen et al., 2012) and a 


\section{India $(n=35) \square$ South_China $(n=18) \quad$ North_China $(n=97)$}
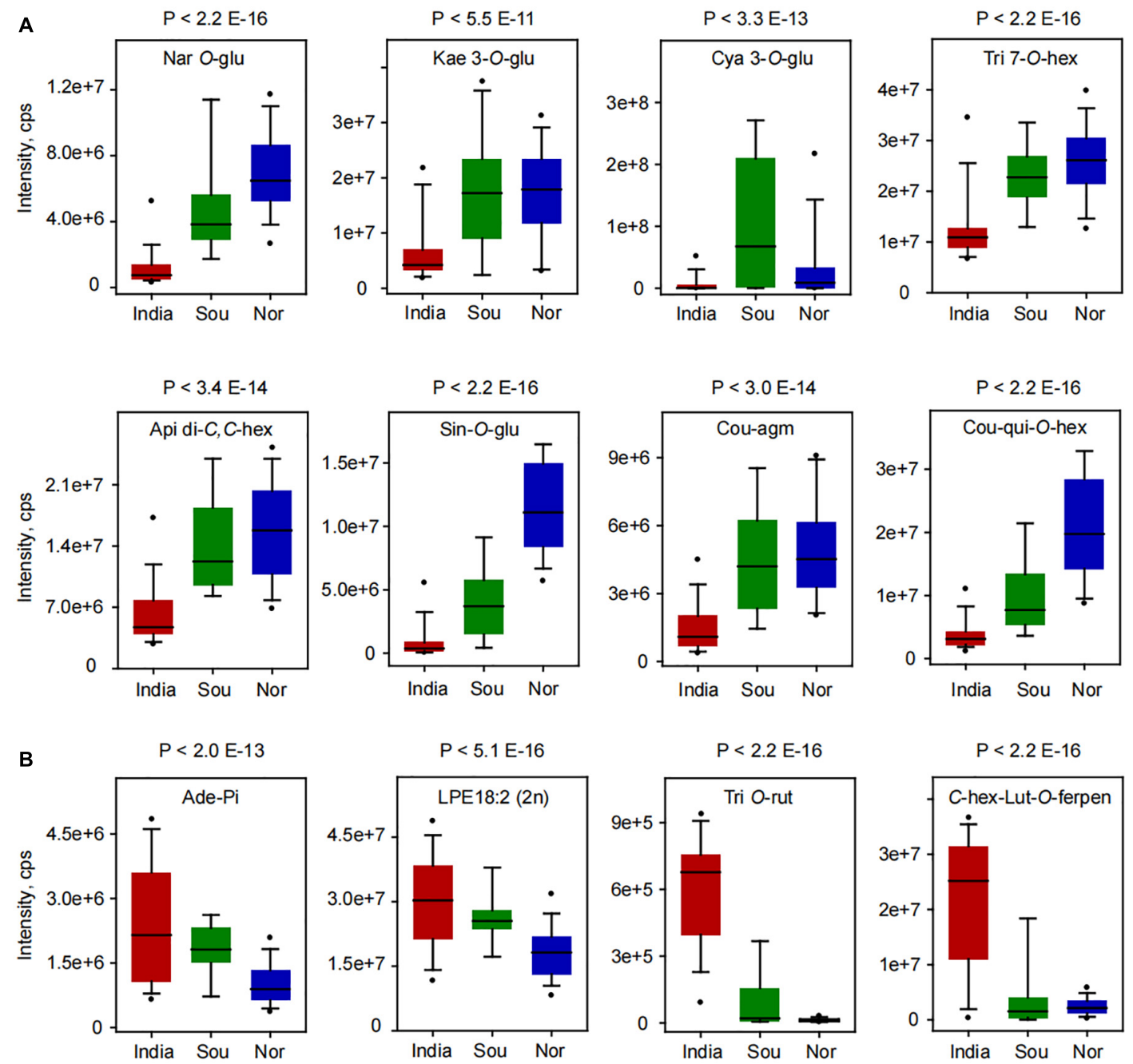

FIGURE 2 | Boxplot for the content of differentially accumulated metabolites among the millets from India, the south of China (Sou), and the north of China (Nor). (A) Significantly higher metabolites were found in millet from northern China. (B) Significantly higher metabolites were found in millet from India. Ade-Pi, adenosine 5'-monophosphate; Api di-C,C-hex, apigenin di-C,C-hexoside; C-hex-Lut-O-ferpen, $C$-hexosyl-luteolin O-feruloylpentoside; Cou-agm, $N^{\prime}$ - $p$-coumaroyl agmatine; Cou-qui-O-hex, 3-O-p-coumaroyl quinic acid O-hexoside; Cya 3-O-glu, cyanidin 3-O-glucoside (Kuromanin); Kae 3-O-glu, kaempferol 3-O-glucoside (Astragalin); LPE18:2 (2n), LysoPE 18:2 (2n isomer); Nar O-glu, naringenin O-glucoside; Sin-O-glu, 1-O-beta-D-glucopyranosyl sinapate; Tri 7-O-hex, tricin 7-O-hexoside; Tri O-rut, tricin O-rutinoside.

final set of 1,963,697 high-quality SNPs were identified by selecting SNPs with missing rates of less than $10 \%$. We then performed mGWAS for the foxtail millet population using these SNPs with a cMLM that results in avoiding fitting transition and saves calculation time. Thereby, we generated a total of 973 lead SNPs for 157 metabolites (Supplementary Table 5), corresponding to 237 quantitative trait loci (Table 1 and Supplementary Tables 6, 7), underlying the genomewide significant threshold $P_{\mathrm{cMLM}}=1.8 \mathrm{E}-06$ after Bonferroni correction. Statistical analysis showed $11.76 \%$ of the metabolites detected had at least one significant association, with an average of 6.2 associations per metabolite (Supplementary Table 7). The significant association for metabolites in different categories was illustrated (Figure 3A), and there were 47 potential mGWAS hot spots, which were mainly located in chromosome 1, 5, and 9, respectively (Figure 3B and Supplementary Table 9). Moreover, we identified that a wide range of metabolites within the same and different categories was co-localized by mGWAS. For example, we observed flavonoids in different subgroups, including anthocyanins, flavonol O-glycosides, and malonylated flavonol $\mathrm{O}$-glycosides, comapped to $43.55 \mathrm{Mb}$ on chromosome 5 and $26.9 \mathrm{Mb}$ on chromosome 7. Chrysoeriol $\mathrm{C}$-hexoside-O-feruloyl-O-pentoside (GZ2812) is an example of a metabolite co-mapping with metabolites in different classes; it comapped with 1-O-p-coumaroyl quinic acid (GZ0548) and 3-O-p-coumaroyl quinic acid O-hexoside (GZ0446), primarily 
TABLE 1 | Summary of genome-wide significant associations identified in the mGWAS.

\begin{tabular}{lc}
\hline Item & Population \\
\hline Number of traits & 157 \\
Number of lead SNPs & 971 \\
Number of loci & 237 \\
SNPs above 20\% of variation & 821 \\
Maximum explained variation (\%) & 60.3 \\
Explained variation per SNP (\%) & 26.0 \\
\hline
\end{tabular}

at $9.3 \mathrm{Mb}$ on chromosome 9 (Supplementary Table 9). These observations implied common genetic regulation of metabolites, mainly phenylpropanoids, in millet.

To identify the potential Indian and Chinese-differentiated signals at the whole-genome level, the $F_{\mathrm{ST}}$ value was calculated. Further scanning the regions by comparing Indian and Northern Chinese accessions identified genomic differential regions totaling $33.64 \mathrm{Mb}$ in length, mainly distributed on chromosomes 1, 3, 6, 7, and 9 (Figure 3C and Supplementary Table 10). When comparing the significant mGWAS loci for the metabolites with the regions, we found that a total of 35 significant loci corresponding to 58 metabolites were overlapped in the Indian-Chinese differential regions (Supplementary Table 11). For example, the mGWAS had significant loci at $43.5 \mathrm{Mb}$ on chromosome 5 for anthocyanis, and flavonol $\mathrm{O}$-glycosides were located at the differentiated regions. These observations further revealed that the phenylpropanoid pathway was specifically selected during both natural selection and artificial domestication of millet.

\section{Functional Interpretation of GWAS for Anthocyanins and Flavonols}

In our results, we found that the accumulation of anthocyanins, such as cyanidin 3-O-glucoside (GZ2192), and flavonol $\mathrm{O}$-glycosides, such as quercetin $\mathrm{O}$-acetylhexoside (GZ0824, precursor substance for anthocyanin synthesis), in Chinese millet is much higher than that of Indian millet according to GWAS analysis, and we found important identical loci related to their accumulation level (Figure 4A). A closer look revealed that the leading SNP 5:43546119 on chromosome 5 was significantly associated with the level of cyanidin 3-O-glucoside ( $P$-value of $3.0 \mathrm{E}-09)$ and quercetin $O$-acetylhexoside $(P$-value of $1.1 \mathrm{E}-09)$, similarly, another leading SNP 7:26896246 on chromosome 7 was significantly associated with the level of cyanidin 3-O-glucoside $(P$-value of 5.7E-09) and quercetin $O$-acetylhexoside $(P$-value of $1.0 \mathrm{E}-09)$. When searching for the candidate genes in the locus on chromosome 5, we found that the Si000845m gene located $0.13 \mathrm{~kb}$ downstream of lead SNP 5:43546119 and was annotated as an anthocyanin regulatory $L c$ protein. Furthermore, when focused on another locus at chromosome 7 , we found the gene Si012401m located $\pm 4.0 \mathrm{~kb}$ upstream of lead SNP 7:26896246 and is also annotated anthocyanin regulatory $L c$ protein (Figure 4A). In a phylogenetic tree of reported MYC transcription factor, Si000845m and Si012401m were tightly clustered in one subclade with a gene, $Z m L c$ (Figure 4B), reported to be involved in responding to low-temperature induction and regulating anthocyanin biosynthesis (Ludwig et al., 1989; Nathalie et al., 2000).

In searching for possible functional polymorphism(s) underlying the natural variation of anthocyanins and flavonol $O$-glycosides, we found a number of highly significant associations between non-synonymous SNPs in Si000845m and Si012401m and the levels of cyanidin 3-O-glucoside (Figure 4C). Among which, one allelic mutation (SNP 5:43550809) in the Si000845m coding region was significantly associated with levels of anthocyanins and flavonol $\mathrm{O}$-glycosides, such as cyanidin 3O-glucoside (Supplementary Table 12). This mutation resulted in significant polarity changes of amino acids between two groups (Allele I and II; Supplementary Table 12). The mean cyanidin 3-O-glucoside content in allele II (G) was significantly higher when compared with that in allele I $(\mathrm{C})$, with $p$-values 2.5E-03 (Figure 4D and Supplementary Table 12). This is consistent with the significant correlation value between distribution differences in millet fields and two group ratios (Figure 4D). Similarly, a significant relationship ( $p$-value of 1.0E-10) was observed between cyanidin 3-O-glucoside content and SNP7:26887726, which caused non-synonymous mutations and in Si012401 $\mathrm{m}$ coding region (Figure 4E and Supplementary Table 12). Furthermore, there was a significant correlation between the two alleles ratio and the cyanidin 3-O-glucoside content of millet in different regions (Figure 4E).

Together, we assigned Si000845m and Si012401m as the candidate MYC transcription factors controlling the biosynthesis of both anthocyanins and flavonol $\mathrm{O}$-glycosides, and we propose that genetic variants within their coding regions might contribute to the natural variation in levels of anthocyanins and flavonol $O$-glycosides in millet. We verified the expression levels of candidate genes in leaves of different genotype materials by using qRT-PCR and found that the expression level of Si012401m has the same trend as the content of cyanidin 3-O-glucoside (GZ2192) (Supplementary Figures 2A,B).

\section{Functional Interpretation of GWAS for Flavone O-Glycosides}

The accumulation of flavone-O-glycosides such as luteolin 7-O-glucoside (GZ0794) and kaempferol 3-O-glucoside (GZ2519) in millet from China was markedly higher than that in India. We found that two flavone-O-glycosides (luteolin 7-O-glucoside and kaempferol 3-O-glucoside) were identified at the same loci based on mGWAS (Figure 5A). The leading SNP3:14349356 on chromosome 3 is significantly associated with the level of luteolin 7-O-glucoside ( $p$-value of $3.0 \mathrm{E}-8$ ) and kaempferol 3-O-glucoside ( $p$-value of $9.1 \mathrm{E}-10)$. We next mined candidate genes in the loci on chromosome 3 , and we found five genes downstream of lead SNP3:14349356 and annotated them as related functions of glycosyltransferase: Si024831m (7.03 kb downstream of SNP3:14349356), Si021855m (10.77 kb downstream of SNP3:14349356), Si021892m (14.54 kb downstream of SNP3:14349356), Si025159m $(21.98 \mathrm{~kb}$ downstream of SNP3:14349356), and Si025028m (25.25 kb downstream of SNP3:14349356). Phylogenetic analysis showed 


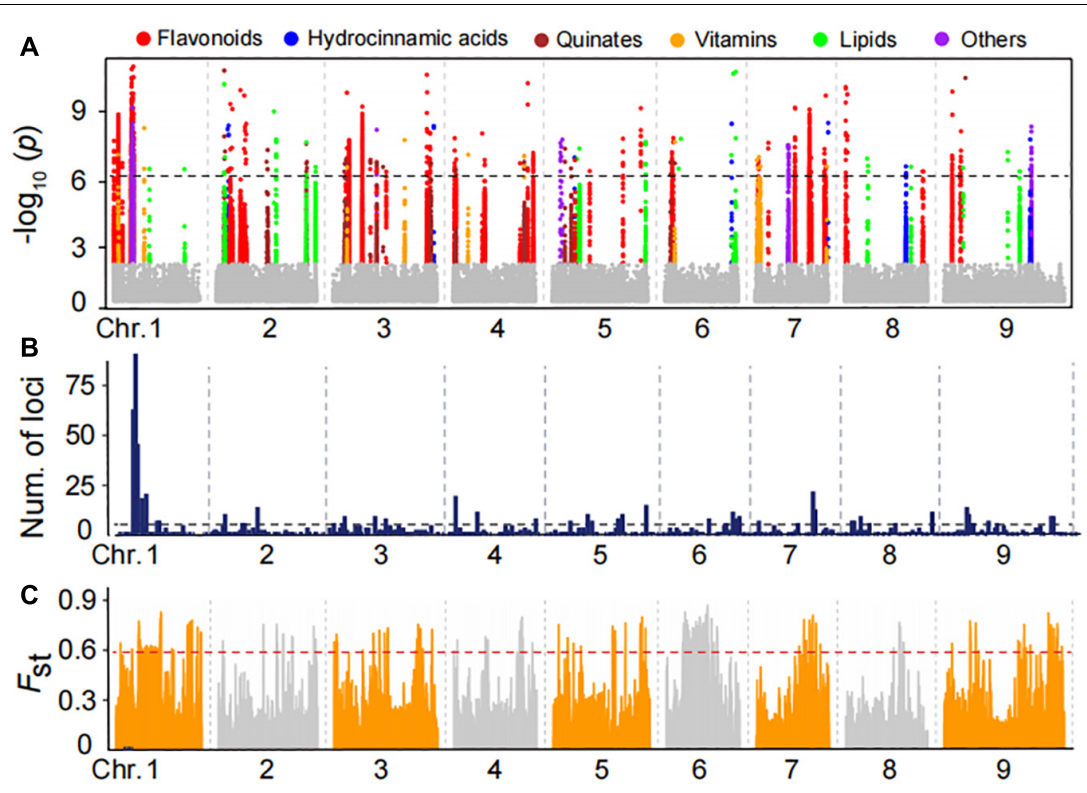

FIGURE 3 | Manhattan plots of mGWAS results and genetic differentiation of mGWAS loci between Indian and Chinese millet. (A) Manhattan plots of mGWAS results with the genetic association for the leaf metabolic features in millet. The strength of association for the metabolites is indicated as the negative logarithm of the $p$-value for the LMM model. All metabolite-SNP associations with P-values below 1.8E-06 are plotted against genome location in intervals of $1 \mathrm{Mb}$.

(B) Distribution of mGWAS signals across the millet genome. (C) Distribution of differentiation ( $F_{S T}$ values) from the comparison of the Indian and northern Chinese millet populations. The regions above the dashed line in the $F_{\mathrm{ST}}$ values distribution are in the $5 \%$ right tail of the empirical distribution $\left(F_{\mathrm{ST}}\right.$ is 0.59$)$.

that five candidate genes have similar sequences to the genes in rice that confer flavone-O-glucosyltransferase (Figure 5B; Peng et al., 2017).

To investigate function variations of candidate genes, we analyzed all SNPs in the full length of the Si024831m. There are 20 SNPs associated with kaempferol 3-O-glucoside content (Figure 5C). Among them, SNP3:14357629 in Si024831m coding region and caused the content of luteolin 7-O-glucoside in millet of the $G$ genotype group to be significantly higher than that of the $\mathrm{T}$ genotype group with a $p$-value of $7.23 \mathrm{E}-18$ (Figure 5D and Supplementary Table 12). Indeed, nonsynonymous SNP3:14358554 are also located in the coding region of the Si024831m, which makes the content of kaempferol 3-Oglucoside in different genotypes of millet differ significantly, with a $p$-value of 1.69E-4 (Figure 5E and Supplementary Table 12). Following this, we further analyzed the allele frequencies of three groups of millet from India, the north of China, and the south of China, and we found, that compared with India, millet from the two regions of China has a higher frequency of alleles with kaempferol 3-O-glucoside (C) and luteolin 7-O-glucoside (G) content and thus has a higher kaempferol 3-O-glucoside and luteolin 7-O-glucoside content (Figures 5D,E).

The expression level of Si024831 $\mathrm{m}$ is highly correlated with the content of luteolin 7-O-glucoside (GZ0794), and its expression level in materials with high content is significantly higher than that in materials with low content $(p$-value $=9.65 \mathrm{E}-4)$ (Supplementary Figures 2C,D). However, there is no difference in the expression level of SiO21892m between high and low kaempferol 3-O-glucoside (GZ2519) content materials $(p$-value $=0.32)($ Supplementary Figures $2 \mathrm{E}, \mathbf{F})$.

\section{DISCUSSION}

\section{Identification and Natural Variation of Metabolites in Millet}

Metabolites can not only reflect plant physiological states; they also play an important role in plant growth and the interaction between plant and environment (Keurentjes, 2009; Treutter, 2009; Saito and Matsuda, 2010; Zhang et al., 2020). Here, 1335 metabolites, including 330 annotated metabolites and 1005 unknown metabolites, were identified and quantified in leaves at the five-leaf stage from 150 accessions. We found 155 annotated metabolites were differentially enriched of millet in three regions of India, the south and north of China, among which were mainly glycosylated forms of flavonoids and Aromatic acylated metabolites. Based on our study, glycosylated forms of flavonoids in the millets were mainly represented by flavone/flavanone mono $C$-hexosides, flavone di-C,C-hexosides, $C$-hexosyl flavone$O$-hexosides, flavone/flavanone/flavonol O-glycosides, flavone $O$-rutinosides, and anthocyanidin $O$-glycosides, coinciding with previous reports in rice and wheat (Moheb et al., 2011; Dong et al., 2014). Glycosylation modification of secondary metabolites is common in plants and has a wide range of important functions, mainly to respond to abiotic stress and help plants to complete recovery; enhance the antioxidant capacity of plants; and ensure the detoxification of harmful compounds in plants (Julien et al., 2016). Aromatic acylated metabolites detected were mainly represented by hydroxycinnamic acids and their $O$-aglycones, phenolamides, hydroxycinnamoyl quinates/shikimates, and $\mathrm{C}$-hexosyl flavone-O-hydroxycinnamoyl hexosides. It is 

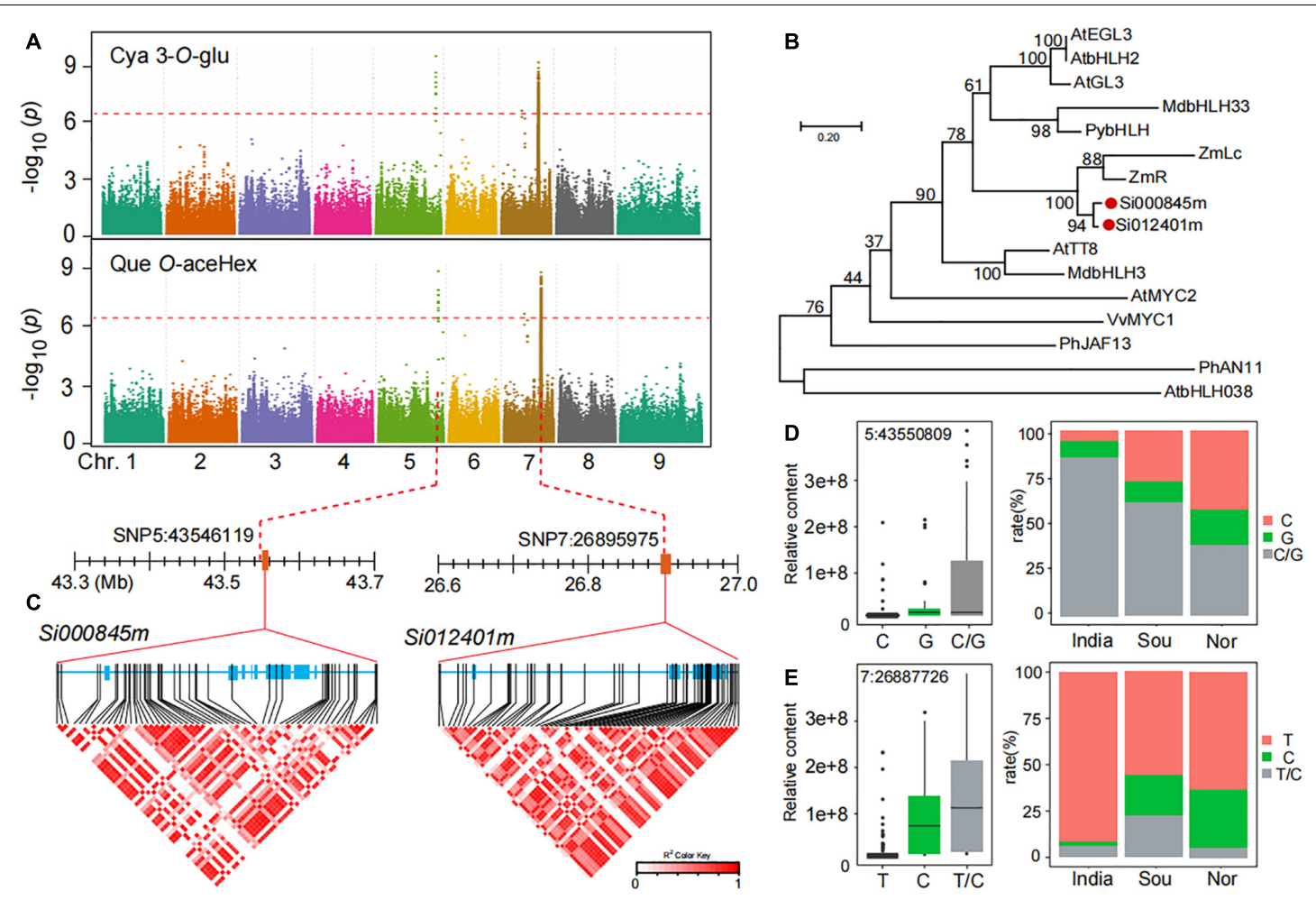

FIGURE 4 | Genome-wide association study of anthocyanins and flavonols in millet. (A) Manhattan plots of mGWAS results of cyanidin 3-O-glucoside (Cya 3-O-Glu) and quercetin $\mathrm{O}$-acetylhexoside (Que $\mathrm{O}$-aceHex) in millet. The strength of association for the metabolites is indicated as the negative logarithm of the $p$-value for the LMM model. All metabolite-SNP associations with $p$-values below 1.8E-06 are plotted against genome location in intervals of $1 \mathrm{Mb}$. (B) An unrooted phylogenetic tree of the MYC protein was constructed as described in Methods. Bootstrap values $>70 \%$ (based on 1000 replications) are indicated at each node (bar: 0.2 amino acid substitutions per site). (C) A representation of pairwise $r^{2}$ values (a measure of LD) among all polymorphic sites in Si000845m and Si012401m, where the color of each box corresponds to the $r^{2}$ value according to the legend. (D,E) Natural variation of nucleotide polymorphisms identified in the sequences of Si000845m and Si012401m and their allelic frequencies in millet from India, south of China (Sou), and north of China (Nor).

well known that the decoration of phytochemicals, such as glycosylation, acylation, methylation, hydroxylation, and prenylation, not only contributes to the diversity of metabolites in plants but is also essential for plant growth and adaptation to the environment (Fang et al., 2018; Wang et al., 2019).

A particular planting environment may cause a specific accumulation of related metabolites in subspecies. The significant natural variation of these decorated phytochemicals especially flavonoids and phenolamides in leaves could be found between the millets from the north of China and India. This difference is usually caused by plants adapting to different geographical environments, thus, combined with the characteristics of the geographical environment, studying the natural variation of metabolites can help us further understand their functions (Wen et al., 2014). Flavonoid aglycones in rice leaf, including flavone mono- $C$-hexosides, flavone $O$-glycosides, and malonylated flavone $O$-hexosides, and phenolamides, including $p$-coumaroyl agmatine/putrescine, showed significantly lower levels in japonica cultivated in the relative higher latitudes (represented by the north of China) than that in indica in the relative lower latitudes (represented by India and the south of China), these metabolites, however, displayed significantly higher levels in the millet from the north of China than that in India, which is important for UV-B protection in plants
(Dong et al., 2014, 2015; Peng et al., 2017). In addition to the UV intensity, the temperature in different latitudes also has obvious differences. The low-temperature environment in high latitudes may cause the active oxygen produced, which leads to frost damage to the crops. As antioxidants, flavonoid aglycone and phenolamide can effectively help crops eliminate freezing damage (Schulz et al., 2015, 2016; Jian et al., 2020; Wong et al., 2020). This may be the reason for the specific enrichment of flavonoid aglycone and phenolamide in northern China. However, the exact mechanism underlying the different patterns of metabolic accumulation in rice and millet requires further investigation.

\section{Natural Variation of Metabolites in Millet Can Be Used in Biomarker}

Metabolites can also be used as biomarkers for subspecies in different planting environments. It has been suggested that flavone $C$-pentosides/hexosides, and malonylated flavonoid $O$-glycosides, are good candidate metabolic markers for distinguishing characteristics of indica and japonica rice varieties (Chen et al., 2013; Dong et al., 2015). Based on the significantly huge variation of metabolites in millet, the differentially accumulated metabolites, such as flavonoids, phenolamides, and hydroxycinnamoyl quinates, could be used as biomarkers for 

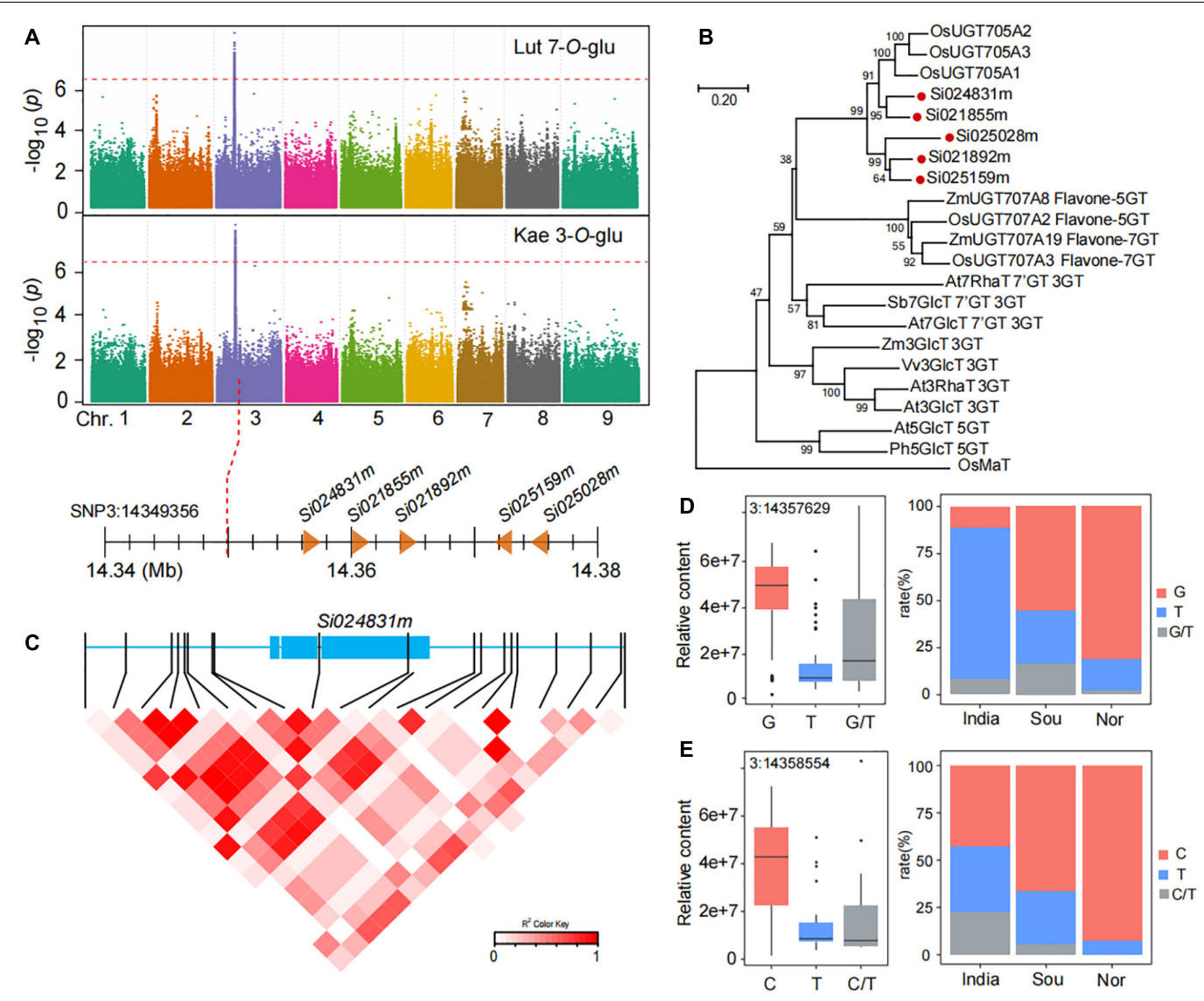

FIGURE 5 | Genome-wide association study of flavone O-glycosides in millet. (A) Manhattan plots of mGWAS results of luteolin 7-O-glucoside (Lut 7-O-Glu), and kaempferol 3-O-glucoside (Kae 3-O-Glu) in millet. The strength of association for the metabolites is indicated as the negative logarithm of the $p$-value for the LMM model. All metabolite-SNP associations with $p$-values below 1.8E-06 are plotted against genome location in intervals of $1 \mathrm{Mb}$. (B) An unrooted phylogenetic tree of the UDP-glycosyltransferase was constructed as described in Methods. Bootstrap values > 70\% (based on 1000 replications) are indicated at each node (bar: 0.2 amino acid substitutions per site). (C) A representation of pairwise $r^{2}$ values (a measure of LD) among all polymorphic sites in Si024831m, where the color of each box corresponds to the $r^{2}$ value according to the legend. (D,E) Natural variation of nucleotide polymorphisms identified in the sequences of Si024831m and their allelic frequencies in millet from India, south of China (Sou), and north of China (Nor).

the discrimination of millet from different areas. For instance, the anthocyanins pelargonidin-3-O-malonyl-malonylhexoside (GZ2423) has obvious differences in the millet leaves of the three regions. Among them, the content of millet leaves in southern China is the highest, which is 596.75 times and 15.48 times that in India and northern China, respectively. Not only that, the content of it in millet leaves in northern China and India is also significantly different, with a multiple of 38.54 (Supplementary Table 4). Hence, Pelargonidin-3-O-malonyl-malonylhexoside can help us determine the source of millet materials. In this study, flavonoid aglycone has obvious differences in millet leaves in different regions and can be used as a marker metabolite to guide planting and breeding.

\section{Homologous Genes of Candidate Genes Are Functionally Conserved in Other Plants}

As a kind of anthocyanin, cyanidin 3-O-glucoside can effectively enhance the tolerance of plants in drought, cold, and high-light environments (Becker et al., 2013; Kovinich et al., 2015). It is formed from dihydroquercetin, catalyzed, and modified by dihydroflavonol 4-reductase (DFR) and anthocyanin synthase (ANS) UDP-glycosyltransferase (UGT) in plants (Nesi et al., 2000; Baudry et al., 2004). In this study, for cyanidin 3$O$-glucoside and quercetin $O$-acetylhexoside, we locate two candidate genes Si000845m and Si012401m on chromosomes 5 and 7 , respectively, and their functional annotated are both anthocyanin regulatory $L c$ protein. Comparative genomics shows that homologous genes from a common ancestor usually have conserved functions, which provides a basis for us to confirm the function of candidate genes in millet (Devos, 2005; Murat et al., 2017; Wang et al., 2019). Two candidate genes are highly homologous to the anthocyanin regulatory Lc protein in maize (Figure 4B), and the gene in maize was transferred into tobacco leaves. Compared with wild tobacco, the anthocyanin content in juvenile leaves in $L c$ transgenic lines strains increased at low temperature, showing a purple phenotype. Transcriptome analysis found that NtDFR 
was only significantly expressed in $L c$-transgenic lines, while the expression of two anthocyanin synthesis related genes $N t A N 2$ and NtANS increased significantly, indicating that NtDFR depends on the regulation of $L c$; again, both NtAN2 and $L c$ can regulate the expression of NtANS, thereby regulating the synthesis of anthocyanins (Huang et al., 2012). This is similar to our research results, which showed the two non-synonymous mutation sites (SNP 7:26887726 and SNP 5:43550809) are highly correlated with cyanidin 3-O-glucoside content in the coding regions of Si012401m and Si000845m. They may serve as sites in response to low-temperature induction and regulate cyanidin 3-O-glucoside content at the post-transcriptional level by affecting gene function. What is more, the distribution of allele frequencies that can increase cyanidin 3-O-glucoside content in millet in India, the south and north of China gradually increases, which is contrary to the temperature changes in these regions.

Luteolin 7-O-glucoside and kaempferol 3-O-glucoside as typical representatives in flavonoid aglycones identified five glycosyltransferase genes Si024831m, Si021855m, Si021892m, Si025159m, and Si025028m within a $200 \mathrm{~kb}$ range (14.356$14.376 \mathrm{Mb}$ ) on chromosome 3, which are homologous to the three glycosyltransferases OsUGT705A1, OsUGT705A2, and OsUGT705A3 in rice, and these three genes in rice are also located in a small interval and have been confirmed to have flavonoid 7-O-glycosyltransferase and flavonoid $3^{\prime}-O$ glycosyltransferase activity (Peng et al., 2017). So Si024831m, SiO21855m, SiO21892m, SiO25159m, and Si025028m in millet may also have the function of transferring glycosyl groups to flavonoids. Correspondingly, the frequency of alleles that increase the content of luteolin 7-O-glucoside and kaempferol 3-Oglucoside is significantly higher in millet in the north of China than in India. The increase in flavonoid aglycones content such as luteolin 7-O-glucoside and kaempferol 3-O-glucoside helps plants withstand low temperatures environment. Metabolomicsbased genome-wide association analysis (mGWAS) has been widely used in Arabidopsis, rice, and maize, deepening our cognition of the genetic basis of these crop metabolomes, greatly promoting the development of functional genomics. Our research fills the gap in the application of mGWAS in millet and paves the way for future dissection of complex physiological traits in millet.

\section{CONCLUSION}

In the present study, LC-MS-based widely targeted metabolic profiling analysis was performed in a wide range of millet accessions. We observed differentially accumulated patterns of metabolites, including flavonoids and phenolamides, between the millets from India and the south and north of China. Moreover, we researched the genetic basis of metabolites by mGWAS analysis found that cyanidin 3-O-glucoside and quercetin $\mathrm{O}$-acetylhexside are concentratedly located at $43.55 \mathrm{Mb}$ on chromosome 5 and $26.9 \mathrm{Mb}$ on chromosome 7 , and two Lc were mined as candidate genes, respectively. However, the signals of luteolin 7-O-glucoside and kaempferol 3-O-glucoside were also detected at $14.36 \mathrm{Mb}$ on chromosome 3 , and five glycosyltransferase genes on this loci were deemed to regulate their content. Then, we used phylogenetic analysis of candidate genes and reported genes to further determine their functions. Our work is the first research to use mGWAS in millet, and it paves the way for future dissection of complex physiological traits in millet.

\section{DATA AVAILABILITY STATEMENT}

The data presented in the study are deposited in the European Variation Archive (EVA) repository, accession number PRJEB45075.

\section{AUTHOR CONTRIBUTIONS}

$\mathrm{ZZ}$ and $\mathrm{XW}$ designed the research. WW and SL supervised this study. WW, XZ, GSh, GSo, WYZ, JP, DW, XL, YZ, TT, and CX participated in the material preparation. JS, WW, FZ, XW, FW, XF, and HL carried out the metabolite analyses. YW, QZ, BW, SL, WQZ, and GF performed the annotation of the metabolites and performed the data analysis. WW and SL discussed the results and wrote the manuscript. All authors contributed to the article and approved the submitted version.

\section{FUNDING}

This research was supported by the following funding sources: the Research and Development Plan "Hybrid Millet Breeding Innovation and Efficient Seed Production Technology Research" of Hebei Province (19226328d), the Science and Technology Research and Development Plan "Hybrid Millet Industry Chain Innovation" of Hebei Province (16226310d), the National Millet Sorghum Industry Technology System Heterosis Utilization Post (cars-06-13.5-a5), the Hebei Province Miscellaneous Grain and Soybean Industry Technology System High-quality Spring Valley Post, and the Zhangjiakou Special Project of Municipal Finance "Collection and Utilization of Hybrid Millet Germplasm Resources and Breeding of New Varieties."

\section{ACKNOWLEDGMENTS}

We thank Yanqiang Gao at Chongqing University for technical support with HPLC and LC-MS/MS analysis.

\section{SUPPLEMENTARY MATERIAL}

The Supplementary Material for this article can be found online at: https://www.frontiersin.org/articles/10.3389/fpls.2021. 665530/full\#supplementary-material 


\section{REFERENCES}

Aidoo, M. K., Bdolach, E., Fait, A., Lazarovitch, N., and Rachmilevitch, S. (2016). Tolerance to high soil temperature in foxtail millet (Setaria italica L.) is related to shoot and root growth and metabolism. Plant Physiol. Biochem. 106, 73-81. doi: 10.1016/j.plaphy.2016.04.038

Alvarez-Rivera, G., Ballesteros-Vivas, D., Parada-Alfonso, F., Ibañez, E., and Cifuentes, A. (2019). Recent applications of high resolution mass spectrometry for the characterization of plant natural products. Trend Anal. Chem. 112, 87-101. doi: 10.1016/j.trac.2019.01.002

Ballesteros-Vivas, D., Álvarez-Rivera, G., Ibáñez, E., Parada-Alfonso, F., and Cifuentes, A. (2019). A multi-analytical platform based on pressurized-liquid extraction, in vitro assays and liquid chromatography/gas chromatography coupled to high resolution mass spectrometry for food by-products valorisation. part 2: characterization of bioactive compounds from goldenberry (Physalis peruviana L.) calyx extracts using hyphenated techniques. J. Chromatogr. A. 1584, 144-154. doi: 10.1016/j.chroma.2018.11.054

Barton, L., Newsome, S. D., Chen, F. H., Wang, H., Guilderson, T. P., and Bettinger, R. L. (2009). Agricultural origins and the isotopic identity of domestication in northern China. Proc. Natl. Acad. Sci. U.S.A. 106, 5523-5528. doi: 10.1073/pnas. 0809960106

Baudry, A., Heim, M. A., Dubreucq, B., Caboche, M., Weisshaar, B., and Lepiniec, L. (2004). TT2, TT8, and TTG1 synergistically specify the expression of BANYULS and proanthocyanidin biosynthesis in Arabidopsis thaliana. Plant J. 39, 366-380. doi: 10.1111/j.1365-313x.2004.02138.x

Becker, C., Kläring, H. P., Kroh, L. W., and Krumbein, A. (2013). Temporary reduction of radiation does not permanently reduce flavonoid glycosides and phenolic acids in red lettuce. Plant Physiol. Biochem. 72, 154-160. doi: 10.1016/ j.plaphy.2013.05.006

Bennetzen, J. L., Schmutz, J., Wang, H., Percifield, R., Hawkins, J., Pontaroli, A. C., et al. (2012). Reference genome sequence of the model plant Setaria. Nat. Biotechnol. 30, 555-561. doi: 10.1038/nbt.2196

Bettinger, R., Barton, L., and Morgan, C. (2010). The origins of food production in North China: a different kind of agricultural revolution. Evol. Anthropol. 19, 9-21. doi: 10.1002/evan.20236

Chen, J., Hu, X., Shi, T. T., Yin, H. R., Sun, D. F., Hao, Y. F., et al. (2020). Metabolite-based genome-wide association study enables dissection of the flavonoid decoration pathway of wheat kernels. Plant Biotechnol. J. 18, 17221735. doi: $10.1111 /$ pbi.13335

Chen, W., Gao, Y. Q., Xie, W. B., Gong, L., Lu, K., Wang, W. S., et al. (2014). Genome-wide association analyses provide genetic and biochemical insights into natural variation in rice metabolism. Nat. Genet. 46, 714-721. doi: 10.1038/ ng. 3007

Chen, W., Gong, L., Guo, Z., Wang, W., Zhang, H., Liu, X., et al. (2013). A novel integrated method for large-scale detection, identification, and quantification of widely targeted metabolites: application in the study of rice metabolomics. Mol Plant. 6, 1769-1780. doi: $10.1093 / \mathrm{mp} / \mathrm{sst} 080$

Devos, K. M. (2005). Updating the 'crop circle'. Curr. Opin. Plant Biol. 8, 155-162. doi: 10.1016/j.pbi.2005.01.005

Dong, X., Chen, W., Wang, W., Zhang, H., Liu, X., and Luo, J. (2014). Comprehensive profiling and natural variation of flavonoids in rice. J. Integr. Plant Biol. 56, 876-886. doi: 10.1111/jipb.12204

Dong, X., Gao, Y., Chen, W., Wang, W., Gong, L., Liu, X., et al. (2015). Spatiotemporal distribution of phenolamides and the genetics of natural variation of hydroxycinnamoyl spermidine in rice. Mol. Plant 8, 111-121. doi: 10.1016/j.molp.2014.11.003

Doust, A. N., Kellogg, E. A., Devos, K. M., and Bennetzen, J. L. (2009). Foxtail millet: a sequence-driven grass model system. Plant Physiol. 149, 137-141. doi: $10.2307 / 25594917$

Fang, C., Fernie, A. R., and Luo, J. (2018). Exploring the diversity of plant metabolism. Trends. Plant Sci. 24, 83-98. doi: 10.1016/j.tplants.2018.09.006

Garriga, M., Retamales, J. B., Romero-Bravo, S., Caligari, P. D., and Lobos, G. A. (2014). Chlorophyll, anthocyanin, and gas exchange changes assessed by spectroradiometry in Fragaria chiloensis under salt stress. J. Integr. Plant Biol. 56, 505-515. doi: 10.1111/jipb.12193

Holsinger, K., and Weir, B. (2009). Genetics in geographically structured populations: defining, estimating and interpreting. Nat. Rev. Genet. 10, 639650. doi: $10.1038 / \mathrm{nrg} 2611$
Huang, Z. A., Zhao, T., Fan, H. J., Wang, N., Zheng, S. S., and Ling, H. Q. (2012). The upregulation of NtAN2 expression at low temperature is required for anthocyanin accumulation in juvenile leaves of Lc-transgenic Tobacco (Nicotiana tabacum L.). J. Genet. Genomics 39, 149-156. doi: 10.1016/j.jgg.2012. 01.007

Jian, H., Xie, L., Wang, Y., Cao, Y., Wan, M., Lv, D., et al. (2020). Characterization of cold stress responses in different rapeseed ecotypes based on metabolomics and transcriptomics analyses. PeerJ 8:e8704. doi: 10.7717/peerj.8704

Julien, L. R., Brigitte, H., Anne, C., Simon, H., and Godfrey, N. (2016). Glycosylation is a major regulator of phenylpropanoid availability and biological activity in plants. Front. Plant Sci. 7:735. doi: 10.3389/fpls.2016.00735

Keurentjes, J. J. (2009). Genetical metabolomics: closing in on phenotypes. Curr. Opin. Plant Biol. 12, 223-230. doi: 10.1016/j.pbi.2008.12.003

Kovinich, N., Kayanja, G., Chanoca, A., Otegui, M. S., and Grotewold, E. (2015). Abiotic stresses induce different localizations of anthocyanins in arabidopsis. Plant Signal. Behav. 10:7. doi: 10.1080/15592324.2015.1027850

Lata, C., Gupta, S., and Prasad, M. (2013). Foxtail millet: a model crop for genetic and genomic studies in bioenergy grasses. Crit. Rev. Biotechnol. 33, 328-343. doi: 10.3109/07388551.2012.716809

Li, S. D., Dong, X. K., Fan, G. Y., Yang, Q. F., Shi, J., Wei, W., et al. (2018). Comprehensive profiling and inheritance patterns of metabolites in foxtail millet. Front. Plant Sci. 9:1716. doi: 10.3389/fpls.2018.01716

Ludwig, S. R., Habera, L. F., Dellaporta, S. L., and Wessler, S. R. (1989). Lc, a member of the maize R gene family responsible for tissue-specific anthocyanin production, encodes a protein similar to transcriptional activators and contains the myc-homology region. Proc. Natl. Acad. Sci. U.S.A. 86, 7092-7096. doi: 10.1073/pnas.86.18.7092

Miki, S., Wada, K. C., and Takeno, K. (2015). A possible role of an anthocyanin filter in low-intensity light stress-induced flowering in perilla frutescens var. crispa. J. Plant Physiol. 175, 157-162. doi: 10.1016/j.jplph.2014. 12.002

Moheb, A., Ibrahim, R. K., Roy, R., and Sarhan, F. (2011). Changes in wheat leaf phenolome in response to cold acclimation. Phytochemistry 72, 2294-2307. doi: 10.1016/j.phytochem.2011.08.021

Murat, F., Armero, A., Pont, C., Klopp, C., and Salse, J. (2017). Reconstructing the genome of the most recent common ancestor of flowering plants. Nat. Genet. 49, 490-496. doi: 10.1038/ng.3813

Nathalie, N., Isabelle, D., Clarisse, J., Georges, P., Michel, C., Loïc, L. (2000). The TT8 gene encodes a basic helix-loop-helix domain protein required for expression of DFR and BAN genes in arabidopsis siliques. Plant Cell 12, 1863-1878. doi: $10.1105 /$ tpc. 12.10 .1863

Nesi, N., Debeaujon, I., Jond, C., Pelletier, G., Caboche, M., and Lepiniec, L. (2000). The TT8 gene encodes a basic helix-loop-helix domain protein required for expression of DFR and BAN genes in Arabidopsis siliques. Plant Cell 12, 1863-1878. doi: 10.1105/tpc.12.10.1863

Peng, M., Shahzad, R., Gul, A., Subthain, H., Shen, S. Q., Lei, L., et al. (2017). Differentially evolved glucosyltransferases determine natural variation of rice flavone accumulation and UV-tolerance. Nat. Commun. 8:1975. doi: 10.1038/ s41467-017-02168- $\mathrm{x}$

Saito, K., and Matsuda, F. (2010). Metabolomics for functional genomics, systems biology, and biotechnology. Annu. Rev. Plant Biol. 61, 463-489. doi: 10.1146/ annurev.arplant.043008.09203

Schulz, E., Tohge, T., Zuther, E., Fernie, A. R., and Hincha, D. K. (2015). Natural variation in flavonol and anthocyanin metabolism during cold acclimation in Arabidopsis thaliana accessions. Plant Cell Environ. 38, 1658-1672. doi: 10. $1111 /$ pce.12518

Schulz, E., Tohge, T., Zuther, E., Fernie, A. R., and Hincha, D. K. (2016). Flavonoids are determinants of freezing tolera and cold acclimation in Arabidopsis thaliana. Sci. Rep. 6:34027. doi: 10.1038/srep34027

Treutter, D. (2009). Managing phenol contents in horticultural crops, why and how? Acta Hortic. 817, 71-78. doi: 10.17660/ActaHortic.2009. 817.4

Wang, S. C., Alseekh, S., Fernie, A. R., and Luo, J. (2019). The structure and function of major plant metabolite modifications. Mol. Plant 12, 899-919. doi: 10.1016/j.molp.2019.06.001

Wang, S. C., Tu, H., Wan, J., Chen, W., Liu, X. Q., Luo, J., et al. (2016). Spatiotemporal distribution and natural variation of metabolites in citrus fruits. Food Chem. 199, 8-17. doi: 10.1016/j.foodchem.2015.11.113 
Wen, W. W., Li, D., Li, X., Gao, Y. Q., Li, W. Q., Li, H. H., et al. (2014). Metabolome-based genome-wide association study of maize kernel leads to novel biochemical insights. Nat. Commun. 5:3438. doi: 10.1038/ncomms4438

Wong, C., Ling, Y. S., Wee, J. L. S., Mujahid, A., and Müller, M. (2020). A comparative UHPLC-Q/TOF-MS-based eco-metabolomics approach reveals temperature adaptation of four Nepenthes. species. Sci. Rep. 10:21861. doi: 10.1038/s41598-020-78873-3

Zeng, X. Q., Yuan, H. J., Dong, X. K., Peng, M., Jing, X. Y., Xu, Q. J., et al. (2020). Genome-wide dissection of co-selected UV-B responsive pathways in the UVB adaptation of qingke. Mol. Plant 13, 112-127. doi: 10.1016/j.molp.2019.1 0.009

Zhang, Z., Tian, C. P., Zhang, Y., Li, C. Z. Y., Li, X., Yu, Q., et al. (2020). Transcriptomic and metabolomic analysis provides insights into anthocyanin and procyanidin accumulation in pear. BMC Plant Biol. 20:129. doi: 10.1186/ s12870-020-02344-0

Conflict of Interest: YW, BW, QZ, CX, TT, JS, WQZ, and HL were employed by the company Wuhan Metware Biotechnology Co., Ltd.
The remaining authors declare that the research was conducted in the absence of any commercial or financial relationships that could be construed as a potential conflict of interest.

Publisher's Note: All claims expressed in this article are solely those of the authors and do not necessarily represent those of their affiliated organizations, or those of the publisher, the editors and the reviewers. Any product that may be evaluated in this article, or claim that may be made by its manufacturer, is not guaranteed or endorsed by the publisher.

Copyright (c) 2021 Wei, Li, Wang, Wang, Fan, Zeng, Zhao, Xu, Zhang, Tang, Feng, Shi, Shi, Zhang, Song, Li, Wang, Zhang, Li, Wang, Zhang, Pei, Wang and Zhao. This is an open-access article distributed under the terms of the Creative Commons Attribution License (CC BY). The use, distribution or reproduction in other forums is permitted, provided the original author(s) and the copyright owner(s) are credited and that the original publication in this journal is cited, in accordance with accepted academic practice. No use, distribution or reproduction is permitted which does not comply with these terms. 